Finally, recent results indicate that the induction of expression of ICOS, a costimulatory molecule related to $\mathrm{CD} 28$, on $\mathrm{CD}_{4}{ }^{+}$ cells correlates with positive responses to cancer immunotherapy in patients with prostate cancer $(19,20)$. The present results (9) suggest that persistent BTLA expression on the surface of $\mathrm{T}$ cells with tumor specificity is a poor prognostic biomarker and that the loss of BTLA expression consequent to cancer immunotherapy may be a positive biomarker of response. The data reported in this issue of the JCI by Derré and coworkers are robust and are likely to provide insight particularly given that they are derived from human studies, which often reveal disease mechanisms not evident in murine studies.

\section{Acknowledgments}

We thank the scientific and clinical team and the patients at the University of Pennsylvania for help and guidance in the development of new cancer immunotherapies. This work was supported in part by NIH grant 5R01CA105216 to C.H. June and NIH training grant support for C.M. Paulos.

Address correspondence to: Chrystal M. Paulos or Carl H. June, Abramson Family Cancer Research Institute and Department of Pathology and Laboratory Medicine, University of Pennsylvania, BRB II/III, Room 554,
421 Curie Boulevard, Philadelphia, Pennsylvania 19104-6160, USA. Phone: (202) 5571868 (C.M. Paulos) and (215) 573-5745 (C.H. June); Fax: (215) 573-8590; E-mail: chrystal. paulos@gmail.com (C.M. Paulos) or cjune@ exchange.upenn.edu (C.H. June).

1. Chen L. Co-inhibitory molecules of the B7-CD28 family in the control of T-cell immunity. Nat Rev Immunol. 2004;4(5):336-347.

2. Greenwald RJ, Freeman GJ, Sharpe AH. The B7 family revisited. Annu Rev Immunol. 2005;23:515-548.

3. Riley JL, June CH. The CD28 family: a T-cell rheostat for therapeutic control of T-cell activation. Blood. 2005;105(1):13-21.

4. Bretscher P, Cohn M. A theory of self-nonself discrimination. Science. 1970;169(950):1042-1049.

5. Brunet JF, et al. A new member of the immunoglobulin superfamily - CTLA-4. Nature. 1987;328(6127):267-270.

6. Watanabe $\mathrm{N}$, et al. BTLA is a lymphocyte inhibitory receptor with similarities to CTLA-4 and PD-1. Nat Immunol. 2003;4(7):670-679.

7. Sedy JR, et al. B and T lymphocyte attenuator regulates $T$ cell activation through interaction with herpesvirus entry mediator. Nat Immunol. 2005;6(1):90-98.

8. Oya Y, et al. Development of autoimmune hepatitis-like disease and production of autoantibodies to nuclear antigens in mice lacking $B$ and $T$ lymphocyte attenuator. Arthritis Rheum. 2008;58(8):2498-2510.

9. Derré L, et al. BTLA mediates inhibition of human tumor-specific $\mathrm{CD}^{+} \mathrm{T}$ cells that can be partially reversed by vaccination. J Clin Invest. 2010;120(1):157-167.

10. Cai G, Freeman GJ. The CD160, BTLA, LIGHT/ HVEM pathway: a bidirectional switch regulating Tcell activation. Immunol Rev. 2009;229(1):244-258.

11. Speiser DE, et al. Rapid and strong human CD8+ $\mathrm{T}$ cell responses to vaccination with peptide, IFA, and CpG oligodeoxynucleotide 7909. J Clin Invest.
2005;115(3):739-746

12. Coley WB. The treatment of malignant tumors by repeated inoculations of erysipelas. With a report of ten original cases. 1893. Clin Orthop Relat Res. 1991;(262):3-11.

13. Paulos CM, et al. Toll-like receptors in tumor immunotherapy. Clin Cancer Res. 2007;13(18 pt 1):5280-5289.

14. Paulos CM, et al. Microbial translocation augments the function of adoptively transferred self/tumorspecific CD8+ T cells via TLR4 signaling. J Clin Invest. 2007;117(8):2197-2204.

15. Apetoh L, et al. Toll-like receptor 4-dependent contribution of the immune system to anticancer chemotherapy and radiotherapy. Nat Med. 2007;13(9):1050-1059.

16. Carpenter EL, et al. Collapse of the CD27+ B-cell compartment associated with systemic plasmacytosis in patients with advanced melanoma and other cancers. Clin Cancer Res. 2009;15(13):4277-4287.

17. Melero I, Martinez-Forero I, Dubrot J, Suarez N, Palazón A, Chen L. Palettes of vaccines and immunostimulatory monoclonal antibodies for combination. Clin Cancer Res. 2009;15(5):1507-1509.

18. Liu X, et al. Cutting edge: a critical role of $B$ and $T$ lymphocyte attenuator in peripheral $\mathrm{T}$ cell tolerance induction. J Immunol. 2009;182(8):4516-4520.

19. Liakou CI, et al. CTLA-4 blockade increases IFNgamma-producing CD4+ICOShi cells to shift the ratio of effector to regulatory $\mathrm{T}$ cells in cancer patients. Proc Natl Acad Sci U S A. 2008;105(39):14987-14992.

20. Chen $\mathrm{H}$, et al. Anti-CTLA-4 therapy results in higher CD4+ICOShi T cell frequency and IFNgamma levels in both nonmalignant and malignant prostate tissues. Proc Natl Acad Sci U S A. 2009;106(8):2729-2734.

21. Paterson A, Vanuri V, Sharpe A. SnapShot: B7/ CD28 costimulation. Cell. 2009;137(5):974-975.

22. Croft M. The evolving crosstalk between co-stimulatory and co-inhibitory receptors: HVEM-BTLA. Trends Immunol. 2005;26(6):292-294.

\title{
Sodium channels gone wild: resurgent current from neuronal and muscle channelopathies
}

\author{
Stephen C. Cannon ${ }^{1}$ and Bruce P. Bean² \\ 1Department of Neurology, University of Texas Southwestern Medical Center, Dallas, Texas, USA. \\ 2Department of Neurobiology, Harvard Medical School, Boston, Massachusetts, USA.
}

\begin{abstract}
Voltage-dependent sodium channels are the central players in the excitability of neurons, cardiac muscle, and skeletal muscle. Hundreds of mutations in sodium channels have been associated with human disease, particularly genetic forms of epilepsy, arrhythmias, myotonia, and periodic paralysis. In this issue of the JCI, Jarecki and colleagues present evidence suggesting that many such mutations alter the gating of sodium channels to produce resurgent sodium current, an unusual form of gating in which sodium channels reopen following an action potential, thus promoting the firing of another action potential (see the related article beginning on page 369). The results of this study suggest a widespread pathophysiological role for this mechanism, previously described to occur normally in only a few types of neurons.
\end{abstract}

Conflict of interest: The authors have declared that no conflict of interest exists.

Citation for this article: J. Clin. Invest. 120:80-83 (2010). doi:10.1172/JCI41340.
Our understanding of channelopathies human disorders arising from mutations of ion channel genes - has gone through several waves of discovery. First, there was the implication that ion channels may play a causal role in disease pathology from the observation of abnormal ionic conductances in muscle biopsied from individuals with myotonia or periodic paralysis, studied using microelectrode recording $(1,2)$. Then came identification of mutations in ion channel genes, made possible by discovery of ion channel gene superfamilies; disease-associated mutations were identified by genome-wide linkage studies or by a candidate gene approach guided by the discovery of aberrant conductances in affected cells (3). This approach enabled the identification of numerous channelopathies in heart, 
A Classic sodium current (hippocampal pyramidal neuron)

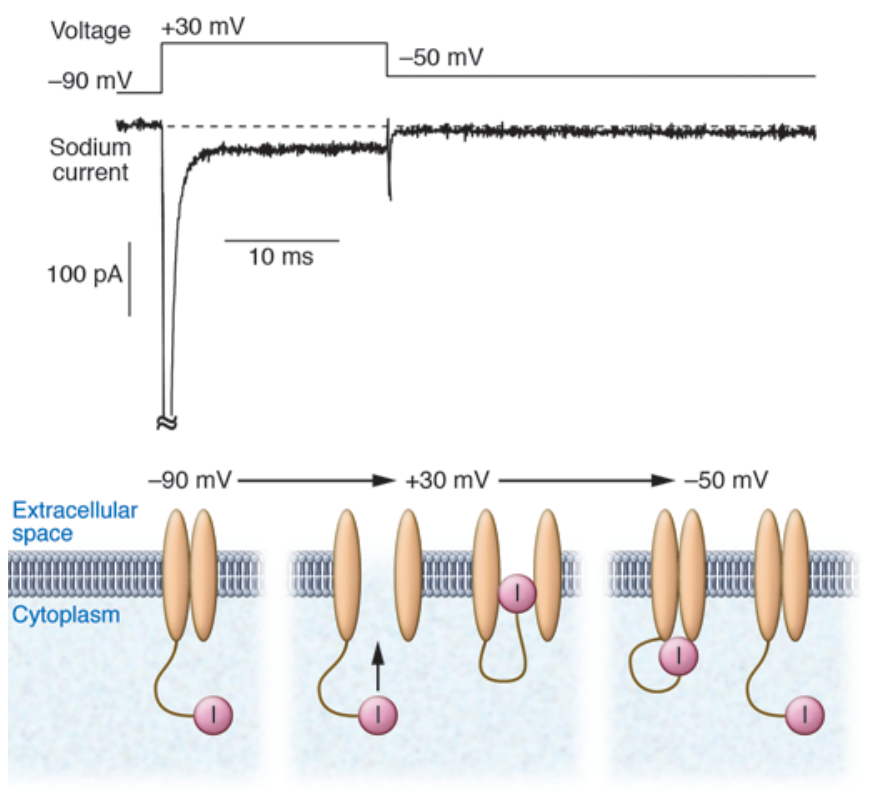

B Resurgent sodium current (cerebellar Purkinje neuron)

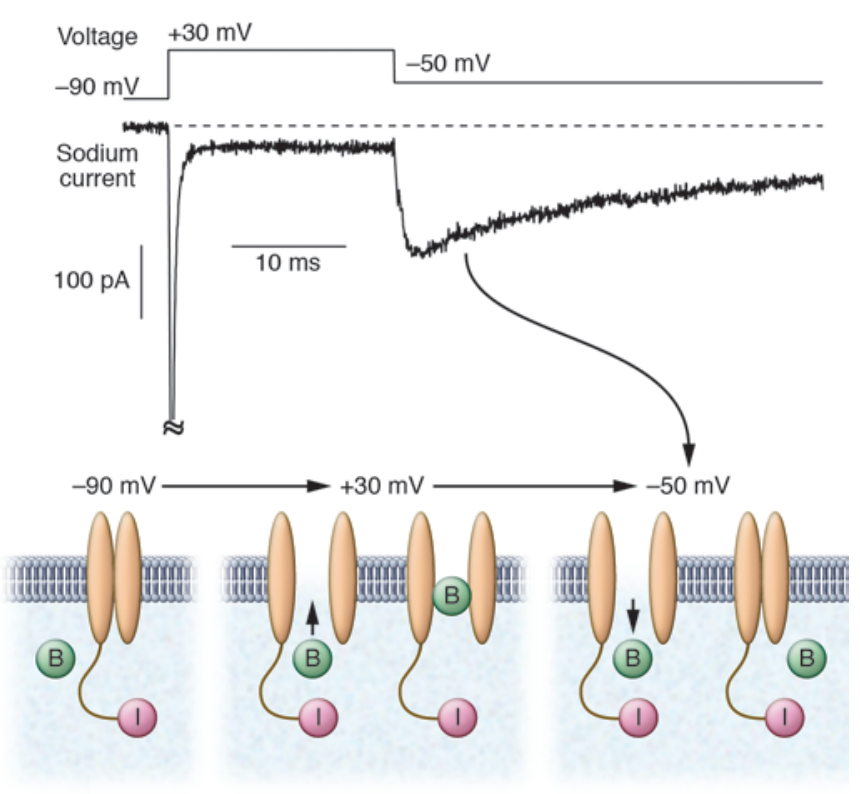

\section{Figure 1}

Conceptual models of sodium channel gating during flow of classic and resurgent sodium current. (A) Conventional sodium current kinetics. Top: Current recorded from a hippocampal CA1 pyramidal neuron in response to indicated voltage sequence. Bottom: Interpretation of channel gating. Channels are closed at $-90 \mathrm{mV}$. Upon depolarization to $+30 \mathrm{mV}$, channels activate, carrying inward sodium current. After a few milliseconds, channels inactivate, corresponding to block of each channel by an intracellular loop of the main channel subunit (particle labeled "I," tethered to channel). Upon repolarization to $-50 \mathrm{mV}$, channels partially recover from inactivation (back to non-inactivated closed state), but no current flows. (B) Sodium current kinetics for sodium channels carrying resurgent current. Top: Current recorded from a cerebellar Purkinje neuron in response to the same voltage sequence shown in A. Bottom: Interpretation of channel gating. In addition to normal inactivation, there is a competing process behaving as if a blocking particle (B), different from the intracellular loop of the main channel subunit, can enter and occlude the channel when it is open, preventing normal inactivation. Upon repolarization to $-50 \mathrm{mV}$, channels occupied by the blocking particle transiently open as the blocking particle exits the channel. The transient opening produces current at $-50 \mathrm{mV}$ (resurgent current). The diagram is oversimplified; in actuality, some channels inactivate normally and do not result in resurgent current. Also, when the blocking particle exits at $-50 \mathrm{mV}$, channels can either return to closed state or enter the normal inactivated state. Current records redrawn with permission from Journal of Neuroscience (19). Schematics of the changing conformation of the channels modified with permission from Journal of Neuroscience (20).

brain, and peripheral nerve (4). The third wave of discovery involved the functional characterization of mutant ion channels $(5,6)$, usually by expression of mutant channels in heterologous expression systems, or, more rarely, by study of mutant channels in native tissue. The study by Jarecki and colleagues in this issue of the JCI (7) uses a clever twist for examining functional effects of ion channel mutations, by expressing cloned sodium channels in native neurons instead of a standard expression system - a procedure that allowed functional characterization of a particular kind of channel gating that has previously been impossible to capture in expression systems derived from nonexcitable cells.

By recording ionic currents from mutant channels heterologously expressed in common expression systems, such as frog oocytes or fibroblast cell lines, much progress has been made in our understanding of how mutations affect channel function. Surprisingly, many disease-associated mutations result in the formation of functional channels in these systems, but with abnormal characteristics. The vast majority of functional defects involve channel gating - the process by which channels open or close in response to membrane potential, ligand binding, stretch, or heat. A broad common theme has emerged: the consequence of mutant channel activity is often depolarization of the cell, through either gain-of-function changes (too much ionic current) for sodium or $\mathrm{Ca}^{2+}$ channels or loss-of-function changes for $\mathrm{K}^{+}$or $\mathrm{Cl}^{-}$channels (5). However, this principle is not absolute, since a number of loss-offunction changes in sodium channels are also known (6). These biophysical measurements of channel gating have been complemented by computational models of cellular excitability that demonstrate, for example, how an observed defect in chan- nel gating is sufficient to trigger abnormal bursts of sustained discharges or to render a cell inexcitable as a result of a persistently depolarized state of refractoriness.

\section{Sodium channel gating}

Voltage-gated sodium channels (VGSCs) carry inward sodium current that underlies action potentials in mammalian neurons, skeletal muscle, and cardiac muscle. VGSCs are closed at normal resting potentials. When the cell membrane is depolarized (made less negatively charged), VGSCs first open (i.e., activate) and then inactivate, closing to a state that cannot be opened by further depolarization. Before being available again for activation, the channels must recover from inactivation. Recovery occurs when the membrane is repolarized to the resting potential. In most cells, recovery from inactivation is electrically silent - no sodium current flows (Figure 1A and ref. 8). However, in some neurons, sodium chan- 

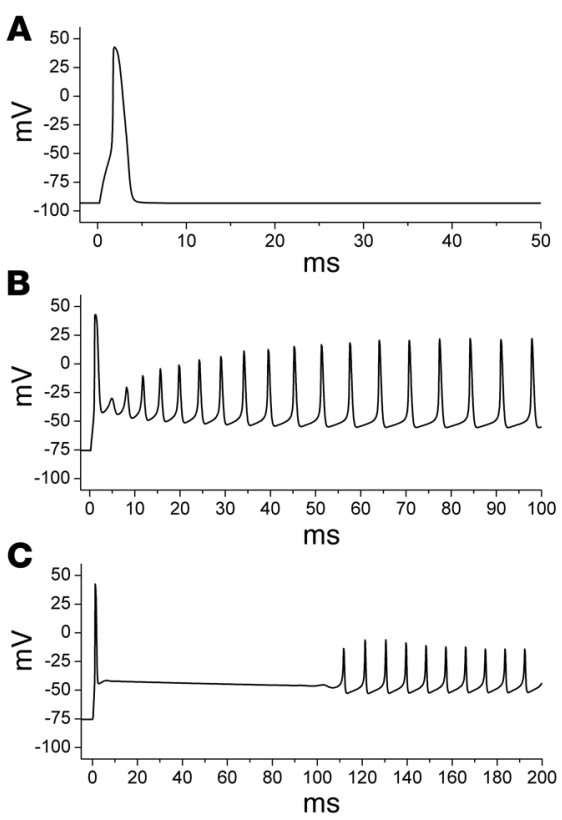

nels open transiently during recovery from inactivation, generating a resurgent sodium current that flows after the membrane is repolarized (Figure 1B and ref. 9). This surge of sodium current provides a depolarizing influence after an action potential, producing a kind of anti-refractory behavior. The mechanism by which resurgent current is generated likely involves an intracellular, positively charged particle that binds to open sodium channels in a voltage-dependent manner, blocking strongly at depolarized voltages but exiting at hyperpolarized voltages $(9,10)$; the exit corresponds to flow of resurgent current at these voltages (Figure 1B). Binding of the particle seems competitive with normal inactivation, which occurs when an intracellular loop of the main channel subunit binds to and occludes the intracellular mouth of the pore (11). In cells with resurgent sodium current, depolarizing current flows as channels recover from inactivation, thereby promoting the firing of a second action potential, and such cells tend to fire bursts of closely spaced action potentials. A small, but growing, list of neurons is known to express resurgent sodium current, including cerebellar Purkinje neurons (9), subthalamic nucleus neurons (12), deep cerebellar nuclei neurons (13), cerebellar granule neurons (13-15), mesencephalic trigeminal motor neurons (16), vestibular nucleus neurons (17), and a subpopulation of primary sensory neurons in dorsal root ganglia (DRG; ref. 18). Resurgent sodium current has not been described in cardiac muscle or skeletal muscle.

\section{Figure 2}

Resurgent sodium current effects on action potential firing in a simulated skeletal muscle fiber. Membrane voltage response to a brief suprathreshold stimulus is shown for 3 different sodium channel configurations. (A) With normal sodium channels, a single action potential is elicited. (B) Simulation of the sodium channel defect in PMC (5-fold slower rate of inactivation compared with wild-type channels, plus $3 \%$ resurgent current), which produces a sustained burst of myotonic after-discharges. (C) Artificial enhancement of the resurgent properties beyond the $3 \%$ used to simulate PMC results in a brief depolarized plateau, which decays to a myotonic burst. Sustained depolarization with action potential failure, as occurs in periodic paralysis, cannot be generated by enhancement of the resurgent sodium current alone.

\section{Testing for sodium channel resurgent properties must be performed in excitable cells}

The exact molecular machinery underlying sodium channel resurgent current is unknown. Grieco and colleagues (10) hypothesized that the blocking particle may be part of a particular accessory subunit, $\beta 4$, but this is still unproven, partly because it has thus far been impossible to reconstitute resurgent gating behavior in heterologous expression systems using cloned channels. In their current study, Jarecki and colleagues overcame this problem by using primary cultures of rat DRG neurons as an expression system for mutant sodium channels (7). From previous work, they knew that a subpopulation of DRG neurons expresses resurgent sodium current normally (18), and thus must possess the necessary intracellular milieu and/or accessory subunits, even if these are not yet well defined. To isolate the sodium current from exogenous sodium channels expressed in the neurons, the authors engineered the channels to lack the binding site for tetrodotoxin (TTX) and used TTX to block current from native sodium channels. With this system, the authors could convincingly identify TTX-resistant resurgent current from the heterologously expressed channels. A limitation of the system is that only approximately $60 \%$ of DRG neurons express resurgent current either normally or after transfection with exogenous channels (presumably reflecting heterogeneity in the cell population), making it necessary to perform many experiments with each mutation to obtain appropriate statistics.

Jarecki and colleagues astutely reasoned that since many disease-associated VGSC mutations slow the process of channel inactivation (5), and the resurgent mechanism appears to compete with normal inactivation (9), such mutations might induce or enhance resurgent current. Their data convincingly demonstrate a proof of principle for this hypothesis (7). They found that mutations associated with painful neuropathy (i.e., in the peripheral neuronal sodium channel Nav1.7 isoform), cardiac arrhythmia (i.e., in the cardiac myocyte sodium channel Nav1.5), or myotonia of skeletal muscle (i.e., in the skeletal muscle sodium channel Nav1.4) all resulted in slowed inactivation and exaggerated resurgent sodium currents when expressed in DRG neurons. The enhancement of resurgent current can be expected to promote high-frequency, repetitive firing in a way that slowing inactivation alone may not; indeed, computer simulations performed as part of their study demonstrate that enhanced resurgent current can, in principle, cause repetitive firing of neurons. Their finding that multiple mutations causing diminished inactivation in multiple sodium channel subtypes can enhance resurgent current is especially exciting because a great many other sodium channelopathies are known to diminish channel inactivation, which suggests that enhancement of resurgent current might occur in many other cases.

\section{Functional consequences in skeletal muscle of altered sodium channels with exaggerated resurgent currents}

How the special features of resurgent sodium current might influence the disease phenotype can be illustrated by modeling of the excitability of skeletal muscle. As pointed out by Jarecki and colleagues (7), an abnormally enhanced resurgent sodium current is expected to promote self-sustained after-discharges in affected muscle fibers, which would manifest clinically as myotonic stiffness caused by involuntary after-contraction. Because the resurgent sodium current is present only transiently upon repolarization, lasting for about 30 or $40 \mathrm{~ms}$, the prediction is that an abnormal resurgent current cannot produce the sustained depolarized shift in the muscle resting potential that underlies an attack of periodic paralysis, such as that predicted by incomplete inactivation without resurgence (5). Figure 2 shows the membrane 
voltage response of a model muscle cell to a brief current stimulus pulse. Normally, a suprathreshold stimulus elicits a single action potential, and the fiber repolarizes to the resting potential (Figure 2A). Simulation of a paramyotonia congenita (PMC) mutant VGSC - for which the kinetics of inactivation are slowed 5 -fold compared with normal sodium channels, and which has a resurgent sodium current of $3 \%$ of the peak current - produces a train of myotonic after-discharges (Figure 2B). If the resurgent component is artificially increased by a 5 -fold increase in the open to open/ blocked rate constant, then the concomitant increase of inward resurgent sodium current does produce a plateau after-depolarization (Figure 2C). But because the resurgent sodium current is transient, the membrane potential eventually repolarizes and breaks into a train of repetitive afterdischarges. This simulation demonstrates that a resurgent sodium current in skeletal muscle will promote myotonia, but is not predicted to cause paralysis, because a transient resurgent current, no matter how large, cannot produce a steady-state depolarized shift in the resting potential.

An important question that remains unanswered is whether mutations that can produce resurgent current in a cell type that allows it (like the DRG neurons used as the expression system) do produce it in the cell types in which the channels are actually present in vivo. For the Nav1.7 channel mutations associated with paroxysmal extreme pain disorder, a rare condition with severe jaw, eye, and rectal pain as well as flushing, the faulty channels are expressed endogenously in DRG neurons, so it seems very likely that the enhanced resurgent channel behavior observed by Jarecki et al. (7) does occur in vivo. However, it is still uncertain whether this is true for mutant cardiac and skeletal muscle channels (Nav1.5 and Nav1.4, respectively), because resurgent sodium current has not been reported in native cardiac or skeletal muscle (although it is also not clear whether appropriate voltage pulse protocols and/or maneuvers to reduce normal inactivation have been used to look for it). It will be crucial to determine whether the cellular milieu in skeletal muscle or heart can support resurgent sodium currents before concluding that resurgent current is a likely disease mechanism for VGSC mutations in these tissues. For mutations in neuronal sodium channels, the expectation is that the mutations will enhance resurgent current only in cell types capable of producing resurgent current under normal circumstances. The full extent of such cell types is not yet known.

Clinically used sodium channel inhibitors, including antiarrhythmics and anticonvulsants, all act by interacting with the gating machinery of sodium channels. Because the molecular machinery of resurgent current is different from that of normal gating, it may well be possible to design or identify drugs capable of selectively reducing resurgent sodium current. If enhanced resurgent current turns out to be a common mechanism for many channelopathies, as suggested by the work by Jarecki and colleagues (7), elucidation of the molecular machinery of this unusual gating mechanism could take on new clinical significance.

Address correspondence to: Stephen C. Cannon, Department of Neurology, UT Southwestern Medical Center, 5223 Harry Hines Blvd., Dallas, Texas 753908813, USA. Phone: (214) 645-6225; Fax: (214) 645-6239; E-mail: steve.cannon@ utsouthwestern.edu.

1. Adrian RH, Bryant $\mathrm{SH}$. On the repetitive discharge in myotonic muscle fibres. J Physiol. 1974;240(2):505-515.

2. Lehmann-Horn F, Rüdel R, Ricker K, Lorkovic H, Dengler R, Hopf HC. Two cases of adynamia episodica hereditaria: in vitro investigation of muscle cell membrane and contraction parameters. Muscle Nerve. 1983;6(2):113-121.

3 . George AL Jr. Inherited disorders of voltage-gated sodium channels. J Clin Invest. 2005;115(8):1990-1999.

4. Lehmann-Horn F, Jurkat-Rott K. Voltage-gated ion channels and hereditary disease. Physiol Rev.
1999;79(4):1317-1372.

5. Cannon SC. Pathomechanisms in channelopathies of skeletal muscle and brain. Annu Rev Neurosci. 2006;29:387-415.

6. Catterall WA, Dib-Hajj S, Meisler MH, Pietrobon D. Inherited neuronal ion channelopathies: new windows on complex neurological diseases. J Neurosci. 2008;28(46):11768-11777.

7. Jarecki BW, Piekarz AD, Jackson JO II, Cummins TR. Human voltage-gated sodium channel mutations that cause inherited neuronal and muscle channelopathies increase resurgent sodium currents. J Clin Invest. 2010;120(1):369-378.

8. Kuo C, Bean BP. Sodium channels must deactivate to recover from inactivation. Neuron. 1994;12(4):819-829.

9. Raman IM, Bean BP. Inactivation and recovery of sodium currents in cerebellar Purkinje neurons: evidence for two mechanisms. Biophys $J$. 2001;80(2):729-737.

10. Grieco TM, Malhotra JD, Chen C, Isom LL, Raman IM. Open-channel block by the cytoplasmic tail of sodium channel beta 4 as a mechanism for resurgent sodium current. Neuron. 2005;45(2):233-244.

11. West JW, Patton DE, Scheuer T, Wang Y, Goldin AL, Catterall WA. A cluster of hydrophobic amino acid residues required for fast $\mathrm{Na}^{+}$-channel inactivation. Proc Natl Acad Sci U S A. 1992;89(22):10910-10914.

12. Do MT, Bean BP. Sodium currents in subthalamic nucleus neurons from Nav1.6-null mice. J Neurophysiol. 2004;92(2):726-733.

13. Afshari FS, et al. Resurgent Na currents in four classes of neurons of the cerebellum. J Neurophysiol. 2004;92(5):2831-2843.

14. Castelli L, Nigro MJ, Magistretti J. Analysis of resurgent sodium-current expression in rat parahippocampal cortices and hippocampal formation. Brain Res. 2007;1163:44-55.

15. Magistretti J, Castelli L, Forti L, D’Angelo E. Kinetic and functional analysis of transient, persistent and resurgent sodium currents in rat cerebellar granule cells in situ: an electrophysiological and modelling study. J Physiol. 2006;573(Pt 1):83-106.

16. Enomoto A, Han JM, Hsiao CF, Chandler SH. Sodium currents in mesencephalic trigeminal neurons from Nav1.6 null mice. J Neurophysiol. 2007;98(2):710-719.

17. Gittis AH, du Lac S. Similar properties of transient, persistent, and resurgent Na currents in GABAergic and non-GABAergic vestibular nucleus neurons. J Neurophysiol. 2008;99(5):2060-2065.

18. Cummins TR, Dib-Hajj SD, Herzog RI, Waxman SG. Nav1.6 channels generate resurgent sodium currents in spinal sensory neurons. FEBS Lett. 2005;579(10):2166-2170.

19. Raman IM, Bean BP. Resurgent sodium current and action potential formation in dissociated cerebellar Purkinje neurons. J Neurosci. 1997;17(12):4517-5426.

20. Grieco TM, Afshari FS, Raman IM. A role for phosporylation in the maintenance of resurgent sodium current in cerebellar Purkinje neurons. J Neurosci. 2002;22(8):3100-3107. 


\title{
New roles for Notch in tuberous sclerosis
}

\author{
Warren S. Pear \\ Department of Pathology and Laboratory Medicine, Abramson Family Cancer Research Institute, University of Pennsylvania, Philadelphia, Pennsylvania, USA.
}

\begin{abstract}
Tuberous sclerosis complex (TSC) is a dominantly inherited disease that is characterized by the growth of multiple benign tumors that are often difficult to treat. TSC is caused by mutations that inactivate the TSC1 or TSC2 genes, which normally function to inhibit activation of mammalian target of rapamycin signaling. In this issue of the JCI, two studies reported by Karbowniczek et al. and Ma et al. link TSC inactivation with activated Notch signaling (see the related articles beginning on pages 93 and 103, respectively). Using a variety of approaches, both studies show that inactivation of TSC leads to Notch1 activation. Furthermore, studies in tumor cells suggest that inhibiting Notch slows growth of the tumor cells. Although much remains to be learned about the precise mechanisms by which TSC loss leads to Notch activation, the newly identified link of TSC to Notch provides the rationale for testing Notch inhibitors in TSC-associated tumors.
\end{abstract}

Tuberous sclerosis complex (TSC) is a dominantly inherited multisystem disease that is characterized by the formation of benign tumors in multiple organs $(1,2)$. The clinical manifestations of TSC are variable but frequently involve the nervous system, as evidenced by the occurrence of seizures and autism, which may, in part, result from benign growths ("tubers") in the cerebral cortex. Patients frequently develop tumors outside of the CNS; the development of renal angiomyolipomas (benign renal neoplasms composed of fat, vascular, and smooth muscle) and pulmonary lymphangioleiomyomatosis (LAM; abnormal proliferation of smooth muscle cells throughout lung interstitium) can lead to severe clinical problems that respond poorly to current treatments. TSC is caused by mutations that inactivate either the TSC1 or TSC2 genes, also known as hamartin and tuberin, respectively (3). TSC1 and TSC2 heterodimerize to inhibit the Ras homolog Rheb (Ras homo$\log$ enriched in brain), which functions to activate the mammalian target of rapamycin (mTOR) complex 1 (TORC1) (4) (Figure 1). Thus, inactivation of TSC1 or TSC2 leads to prolonged Rheb signaling and activation of the rapamycin-sensitive TORC $1 \mathrm{arm}$ of the mTOR signaling pathway. mTOR integrates a variety of signals induced by growth factor stimulation, hypoxia, and nutrient availability to control cell cycle, nutrient uptake, and transcription and translational control (5). Important downstream targets of mTOR are

Conflict of interest: W.S. Pear owns stock in Pfizer Inc.

Citation for this article: J. Clin. Invest. 120:84-87 (2010). doi:10.1172/JCI41897. ribosomal protein $\mathrm{S} 6$ kinase polypeptide 1 (S6K1) and eukaryotic translation initiation factor $4 \mathrm{e}$-binding protein 1 (4E-BP1) that regulate ribosome recruitment and protein translation. mTOR is activated in a wide variety of human tumors and is considered to be an excellent target for anticancer therapy (6). The identification of the MTOR pathway as a major player in the pathogenesis of TSC suggested that specific inhibition of this pathway may substantially benefit TSC patients (7). The data are sobering thus far, as treatment of TSC patients with the mTOR inhibitor sirolimus has induced only mild, transient improvement (8). These data suggest that better and/or additional therapeutic agents are needed to treat TSC. In this issue of the JCI, Karbowniczek et al. (9) and Ma et al. (10) report that TSC loss is associated with upregulation of the Notch signaling pathway. Therapeutic Notch inhibitors have already been developed, and related findings suggest that these inhibitors may be beneficial for the treatment of TSC.

TSC loss promotes Rheb-dependent Notch activation in flies, rodents, and humans

Inappropriate expression of lineage markers is a frequent characteristic of TSC tumors; CNS tubers express both neuronal and glial lineage markers, and both LAM and renal angiomyolipomas express smooth muscle and melanocytic markers. Moreover, cells within the clonal renal angiomyolipomas exhibit developmental plasticity, with the capacity to develop into fat, smooth muscle, and vascular lineage cells (11). These obser- vations served as the initial impetus for both Karbowniczek et al. (9) and Ma et al. (10) to explore the potential role of Notch signaling in this process, as Notch is an important arbiter of cell fate decisions (12). Using different model systems and approaches, both groups came to the same conclusion: TSC loss leads to Notch activation. Karbowniczek and coworkers (9) took advantage of the evolutionary conservation of the TSC pathway to study the function of TSC signals in the well-described differentiation of the sensory organ precursor (SOP) cell into the external sensory organ (ESO) of the fruit fly Drosophila melanogaster (13) (Figure 2A). TSC signaling is conserved from Drosophila to humans; for example, the Drosophila orthologs of the mammalian tuberous sclerosis proteins, dTSC1 and dTSC2, function downstream of the insulin receptor to regulate mTOR signals and cell size (14). Differentiation of the SOP cell into the ESO results from multiple asymmetric divisions that depend on differential regulation of Notch signaling to ultimately give rise to the differentiated ESO, which is comprised of an external bristle cell and socket cell as well as an internal sensory neuron and sheath cell (Figure 2A) (15). This process begins when the primary progenitor (pI) cell undergoes an asymmetric cell division to give rise to an anterior $\mathrm{PIIb}$ cell and a posterior pIIa cell. The Notch inhibitor Numb is asymmetrically distributed to the pIIb cell, such that Notch signaling persists in the pIIa cell, whereas it is inhibited in the pIIb cell. In the absence of Notch signaling, two pIIb cells are produced, whereas constitutive Notch signals lead to the production of two pIIa cells at the expense of pIIb differentiation. The latter results in duplication of the external bristle and socket cells, causing the appearance of extra microbristles on the flies. Karbowniczek et al. generated flies with loss-of-function TSC alleles and found that the SOP differentiation phenotypes were similar to Notch gain-of-function phenotypes (i.e., duplication of the pIIa cell resulting in extra microbristles) (Figure 2B). Furthermore, overexpression of Rheb recapitulated these phenotypes, 